the tables of these satellites should be brought to a high degree of perfection; and as, according to the opinion of the most distinguished mathematicians, the observation of all the phenomena which are presented by one of these satellites in superior or inferior conjunction is the best means of determining certain elements of the theory of the satellites of Jupiter, it was useful to give in the collection of ephemerides not only the epochs of the eclipses, but also those of the contact of the shadow of the satellite with the planet. Tables for the observation of the satellites at the time of their maximum elongation would also be very desirable.

From the mariners' point of view, for whom the moon is the principal heavenly body, the positions of the moon calculated for noon and midnight of every day would be insufficient on account of the considerable proper movement of our satellite. To obtain the longitude of a place by means of the observation of the passage across the meridian of one of the limbs, there would be required an exce'ssively laborious calculation; the use of that method, however convenient, was then illusory. It was necessary to give the right ascension and the declination for every hour of the day, for the purpose of avoiding the employment of second differences except in cases where very great precision was sought for.

Then, when accurate tables of the movements of the planets were obtained, it was useful to add to the distances of the moon from the sun and from the stars, the distances of that body from the principal planets, the observation of which is more convenient and more certain than that of its distances from the stars.

But it was necessary to consider not only astronomers in observatories and sailors on board their ships, it was useful to enable astronomers on an expedition, and sailors when in a foreign harbour, and also geographers, to obtain the geographical co-ordinates of their station with ease and accuracy. From this point of view the method known as that of the Lunar Culminations holds the first rank, a method to which a beautiful work by Nicolaï* gave a capital importance. The learned director of the Observatory of Mannheim showed with what facility the observations of the passage of the moon combined with those of a certain number of stars, called "stars of the moon," bordering on its parallel, and passing the meridian a little before or a little after (halfan-hour at the most), could give, sufficiently approximately, the difference of the longitudes of two places, even with a meridian instrument which was not perfect. On the other hand, Bessel and Hansen had given simple methods for calculating the horary movement of the moon. To apply this method of lunar culminations, it was then necessary to choose "stars of the moon," and to publish their positions cvery year, day by day, at the same time as those of the moon at the moment of its passing the meridian. This addition had, moreover, this advantage, that by indicating by an asterisk the stars comprehended between $4^{\circ}$ and $14^{\circ}$ of declination, the observers of the two hemispheres would have the elements most useful for improving continuously (d'une façon continue) the value of the lunar parallax. The phenomenon of the occultation of the stars of the moon offers, besides, an excellent means of determining longitudes. It was then important thus to calculate in advance and to publish all the elements likely to serve for predicting all the occultations in a given place, for the purpose of rendering the employment of this method easy to the navigator.

Finally it was indispensable, as well for the astronomical operations of observatories as for those connected with an astronomical or a geodetic expedition, that the collection of ephemerides should contain, for epochs sufficiently close to permit calculation for intermediate dates

* "Uber die Methocle, längen durch Rectascensions-Differenzen gewählten Vergleichsterne vom Monde zu bestimmen" (Astronomische wählten Vergleichsterne vom
Nachrichten for 1823 and 1824 .) by simple proportion, the apparent positions of a very large number of stars of the greatest magnitude, and distributed both in the north and south hemispheres. It was useful, moreover, to join to this catalogue the values for very close epochs of the constants of Bessel, which enable one to pass from the mean position of a star at the commencement of the year to its apparent position on any day whatever.

For the principal circumpolars, $a$ and $\delta$ Ursæ Minoris, the importance of which is so great in determining the various constants of a meridian instrument, and whose apparent positions vary much more rapidly than those of stars at a distance from the pole,-the apparent positions ought to be given every day.

Such is, with the exception of a few unimportant details, the list of reforms which the general opinion of astronomers demanded in England and Germany.

(To be continued.)

\section{ON THE SECONDARY WAVES IN THE SPHYGMOGRAPH TRACE}

$\mathrm{N}$ a letter printed in this journal a short time ago (vol. viii. p. 464), Dr. Galabin refers to a paper which has been since published in the Fournal of Anatomy and Physiology (No. XII. p. I), for a fuller account of his views as to the theory of the pulse, of which we gave a short notice and criticism in a former number (vol. viii. p. 330). This second and more detailed description calls for further remark, especially as the author has found reason somewhat to modify his opinion on one important point.

As is well known, the sphygmograph trace of a pulse beat (see Fig. I) consists of a primary rapid rise, followed by a more gxadual fall, broken by a considerable undulation, termed the dicrotic wave, which varies in its distance from the next primary rise according to the rapidity of the pulse. Between the primary and the dicrotic rises in the trace, the descending curve is sometimes interrupted by another small undulation termed the "tidal" wave, by Mr. Mahomed, though the name predicrotic is better, as it does not involve any theoretical conceptions. It is the development in the trace of these predicrotic and dicrotic waves that Dr. Galabin discusses and his explanation of the former is the fol lowing.-The separation of the primary and tidal (predicrotic) waves is due to an oscillation in the Sphygmograph, caused by the inertia of the instrument. In some cases the lever may be separated slightly from the knife-edge on which it rests, but generally the oscillation takes place in the instrument as a whole, and it may be followed by others in a descending series. With reference to this interpretation, it may be first remarked that it seems almost impossible that the whole sphygmograph should acquire a momentum in each pulsation, for it should be so adjusted on the arm that no part except the tip of the spring is in any way in contact with the artery, and when such is the case it is difficult to conceive of any shock being communicated to the whole. Again, any sudden upward impulse given to the instrument itself would be attended with a descent in the trace, for as the lever is only attached at one end, and there only on points, its pen would be slow to participate in the general movement of the framework, and would not rise so rapidly as the recording paper. The momentum acquired by the lever is a different thing. Marey and Sanderson have both shown that the primary rise in the trace may be attended with a sudden sharppoinied wave, in the production of which the lever leaves the knife-edge on which it rests, returning to it after a very short excursion. To prevent the excessive development of this imperfection Marey has employed a small secondary spring to depress the lever; this spring Dr. 
Galabin persists in not employing, because he thinksthough the evidence he brings forward on the subject is extremely small - that it increases the number of minor vibratory undulations. Nothing of the kind, however, is the case. Nearly all properly-taken tracings from the pulse in health present, if there is a secondary spring ernployed, no percussion wave at all; and when it is present the true predicrotic wave is quite independent, as may be seen in Fig. 2, which is from a powerful, healthy pulse of 44 a minute, in which the rise $a$ is the percussion, $b$ the primary, $c$ the predicrotic, and $d$ the dicrotic wave. This true predicrotic wavel varies in development with

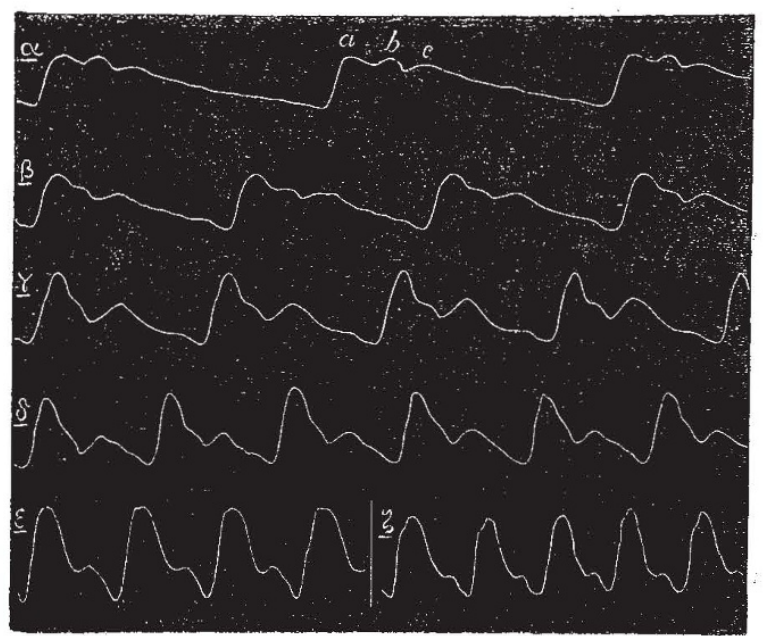

Fig. x. - Sphygmograph tracings of bea thy pulses, drawn to one scale, wit' rates between 44 and $I_{7} \mathrm{O}$ a minute. They read from left to right.

different pulse rates, being much more conspicuous in very slow pulses, and entirely absent in very quick ones, in which last a slight percussion wave is frequently found (see Fig. I). Dr. Sanderson has previously described these two waves as co-existing, and he is undoubtedly right, as any who have had any considerable experience in Sphygmography in health will agree. It is Dr. Galabin who is in error, and it is but little compliment to other workers in the same field even to suppose that they have been sufficiently simple-minded to study and describe as physiological phenomena, instrumental errors so uncomplicated in origin and so readily comprehended. The

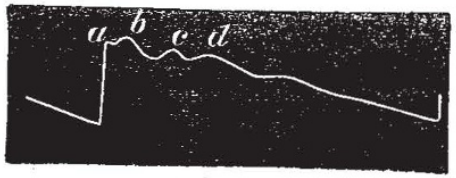

FIG. 2.-A tracing of a healthy pulse beating 44 a minute.

chief argument he brings forward in favour of his explanation is that by placing a weight on the tever at different parts, and so altering its moment of inertia, the length of the predicrotic wave is varied. That the percussion wave which is developed when no secondary spring is employed is so affected, no one will doubt, because the resistance of the pen is less significant when the lever is heavy than when it is light, and therefore the wave is of shorter duration when it is weighted. This wave, however, is even then of such considerable length that it has not ceased before the true predicrotic wave has commenced, and it therefore disguises the true nature of the trace. It is, therefore, only when the secondary spring is employed that a proper trace can be obtained; because then only is it possible to see the full extent of the true predicrotic wave, uncomplicated by the superposition of the extraneous percussion wave. The latter does not appear as an extra element of the curve, but entirely disguises its true nature, on account of its being developed quite independently, when the lever is no longer in connection with the rest of the instrument, and therefore unaffected by whatever change may be occurring in the artery.

The cause of this predicrotic wave, which Marey gives of the similar one that appears in the hæmadromometer trace (Fig. 3, $\beta$ ) though considered by Dr. Galabin scarcely worthy of refutation, is supported by a large number of facts, especially by the hæmadromometer trace itself (Fig. 3, $\alpha, \beta$ ). Its commencing in the radial artery as well as the carotid, at the moment of closure of the aortic valve, is also strongly in favour of the supposition that it is of shock origin; and that a shock may be transmitted through a column of fluid, which Dr. Galabin and some others seem to doubt, can be easily proved by suddenly closing an ordinary tap through which a large volume of water is passing, whereupon several oscillations of the retained liquid occur, producing a series of blows against the tap and perhaps the side of the tube, which are heard without difficulty.

The hæmadromometer trace (Fig. 3) shows also how completely the dicrotic wave is the result of the closure of the aortic valve, as Dr. Galabin also thought

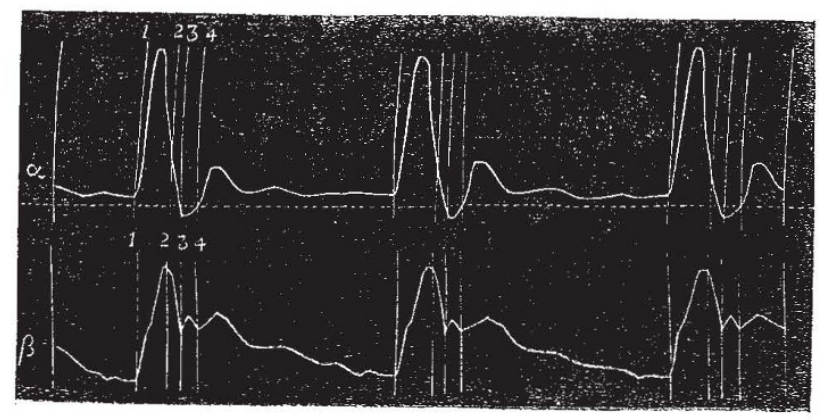

FIG. 3.-Hæmadromograph trace rom the carotid. a, Curve oi direction and force of blood current, all above the dotted line indicating an onward and all below, a heartward stream. $\beta$, Simultaneous sphygmograph trace.

in his earlier paper; but in his second he attributes it to the oscillatory result of the inertia of the arterial walls, and the lateral momentum acquired by the blood. The mass of the arterial walls, and the lateral movement of the blood during distension are so slight, that neither are in any way competent to explain a movement so constant and so considerable as the dicrotic wave, especially when one so much more reasonable is to be obtained as the result of the valve closure. At all events no theory can be considered at all satisfactory which does not explain, in oneway or another, the hæma. dromometer trace, which is one of the foundations of arterial dynamics, and has been verified in all its details by Dr. Lortet of Lyons. Neither Dr. Galabin's theory, nor that of Mr. Mahomed, can be said in any way to take cognizance of the facts which it discloses, and they are incapable of doing so, therefore they must be considered inaccurate. Both these authors complicate their results by arguing from the analogy of a schema or model of the circulation constructed with elastic tubes; the arteries, however, are not simple elastic tubes, but tubes cut in elastic solids, being surrounded on all sides by yielding tissues, and they are not therefore comparable with tubes experimented on in air, and will not allow of comparative deductions being drawn from them.*

\footnotetext{
* The blocks for Figs. I and IIr. are kindly lent by Prof, Humphry,
} 